\title{
Theoretical Study on Separation of Nonmetallic Inclusion Particles from a Hollow Cylindrical Melt in Alternating Electromagnetic Field
}

\author{
Ke LI, Da SHU, Jun WANG, Zhaohui DU, Baode SUN and Yaohe ZHOU \\ The State Key Laboratory of Metal Matrix Composites, Shanghai Jiao Tong University, Shanghai 200030 China. \\ E-mail: keli@sjtu.edu.cn
}

(Received on August 25, 2003; accepted in final form on December 1, 2003)

\begin{abstract}
Kinetic analysis was made on the electromagnetic separation of nonmetallic inclusions from hollow cylindrical melt in an alternating electromagnetic field. Effects of various processing parameters such as magnetic flux density $B_{e}$, imposing time $t$, particle diameter $d_{p}$, the nondimensional parameters $r_{1} / \delta$ and $\lambda$ on the removal efficiency $\eta$ were analyzed theoretically. It is found that higher removal efficiency can be obtained in the hollow cylindrical melt than in the solid cylinder case under the same conditions. Generally, in order to obtain higher removal efficiency, the value of various parameters should be selected properly in hollow cylindrical melt case. For the inclusion particles with diameter larger than $5 \mu \mathrm{m}$, the removal efficiency can exceed 90 pct in the hollow cylindrical case with optimal processing parameters.
\end{abstract}

KEY WORDS: electromagnetic separation; solid cylindrical melt; hollow cylindrical melt; inclusions; separation efficiency.

\section{Introduction}

It is well known that nonmetallic inclusions in the alloy not only form porosity, but also result in stress concentration, low plasticity and low ductility. ${ }^{1)}$ Traditional processes, such as gravity sedimentation/flotation, degassing, flux refining, filtration, can hardly meet the cleanliness levels demanded in most applications due to their low efficiency to remove micrometer sized inclusions and contamination to the environment. ${ }^{2)}$ Thus, a clean, effective and continuous cleaning process has become of great importance to meet the increasing demand for high quality alloy.

Early in the 1980s, the principle of applying electromagnetic forces to inclusion separation has been proposed by $\mathrm{P}$. Marty et al. ${ }^{3)}$ In recent years, the applications of electromagnetic separation techniques with different means of magnetic field generation have been widely studied. Among those techniques, the alternating electromagnetic field method shows great advantages because it does not need loop circle for the imposed current and has no contamination problem on the metal caused by introduction of an electrode. V. M. Korovin ${ }^{4,5)}$ deduced the theory of separating nonmetallic inclusions in the melt by using alternating magnetic field induced in an infinitely long solenoid theoretically. D. Shu ${ }^{6)}$ studied the electromagnetic force exerted on the particles in a solid cylindrical melt and the relation between various processing parameters and the removal efficiency theoretically. In previous works ${ }^{7,8)}$ the effective values of $\eta$ were measured during electromagnetic separation process of aluminum melts, which matches well with the results of theoretical analysis. The above works mainly fo- cused on the cylindrical melt placed in a circular pipe. However, the removal efficiency of inclusion particles was insignificant because most of the particles are located in the central area far from the skin depth layer and less influenced by the magnetic field.

In this paper, the formula of calculating the electromagnetic force imposed on a unit volume particle in the hollow cylindrical melt was derived theoretically. Effects of various parameters such as magnetic flux density $B_{e}$, imposing time $t$, particle diameter $d_{p}$, the nondimensional parameters $r_{1} / \delta$ and $\lambda$ on the terminal migrating velocity and the values of $\eta$ in the hollow cylindrical melt were analyzed and discussed by comparing with solid cylindrical melt case.

\section{Theory in the Solid Cylindrical Melt Case}

In the electromagnetic separating process, eddy current is induced in the solid cylindrical melt which is placed in a pipe surrounded by an alternating electromagnetic field, as shown in Fig. 1. And the interaction of the induced eddy current with the associated magnetic field produces electromagnetic pinch force $\mathbf{f}_{r}$ on the melt:

$$
\mathbf{f}_{r}=\mathbf{J} \times \mathbf{B}
$$

where $\mathbf{f}_{r}$ is the electromagnetic force acting on a unit volume of the melt, $\mathbf{J}$ is the induced electric current density vector and $\mathbf{B}$ is the magnetic field intensity vector, respectively.

Due to the different electrical conductivity between the nonmetallic inclusion particle and the melt, the particle experiences an electromagnetic force $\mathbf{f}_{p r}$ in the opposite direc- 


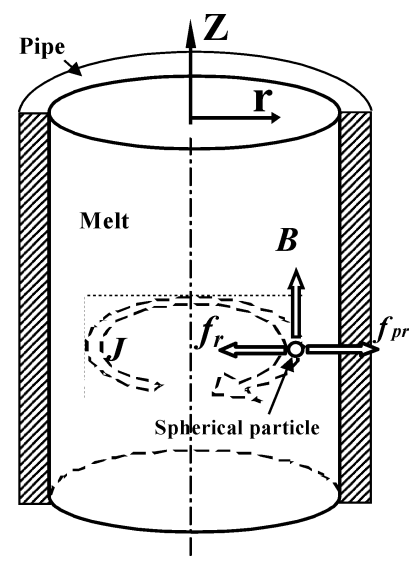

Fig. 1. Application of alternating electromagnetic field on melt cleaning.

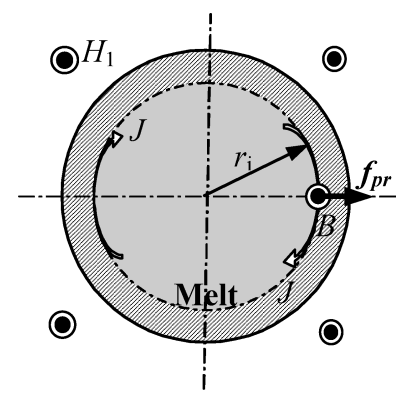

Fig. 2. Electromagnetic force exerted on the cross profile of solid cylindrical melt.

tion to the pinch force, migrating to the outside wall of the pipe and being captured there. For a nonconductive spherical particle, $\mathbf{f}_{p r}$ can be expressed by ${ }^{9,10)}$ :

$$
\mathbf{f}_{p r}=-\frac{3}{4} \frac{\pi d_{p}^{3}}{6} \mathbf{f}_{r}
$$

where $d_{\mathrm{p}}$ is the diameter of the particle, the minus designates that the direction of $\mathbf{f}_{p r}$ is opposite to that of $\mathbf{f}_{r}$.

The root-mean-square value of the electromagnetic pinch force can be expressed by ${ }^{11)}$.

$$
f_{r}=\frac{1}{2} \operatorname{Re}\left(\dot{\mathbf{J}}_{r} \times \mu_{e} \dot{\mathbf{H}}_{r}^{*}\right)
$$

where $f_{r}$ is the root-mean-square value of the electromagnetic pinch force, Re is the real part of the complex number and $\mu_{e}$ is magnetic permeability of the melt. $\dot{\mathbf{j}}_{r}$ represents the complex vector of the induced current and $\dot{\mathbf{H}}_{r}^{*}$ represents the conjugate complex vector of the applied magnetic field strength, respectively.

Figure 2 is a schematic view of the electromagnetic force exerted on the transversal profile of the solid cylindrical melt. In this case, by solving the following diffusion equation ${ }^{12)}$ :

$$
\frac{d^{2} H}{d r^{2}}+\frac{1}{r} \frac{d H}{d r}-\alpha^{2} H=0
$$

where $H$ is the applied magnetic intensity, $\alpha=(1+i) / \delta$, the skin depth $\delta=\sqrt{1 / \pi \mu_{e} \sigma_{f} f}, \sigma_{f}$ is the electrical conductivity

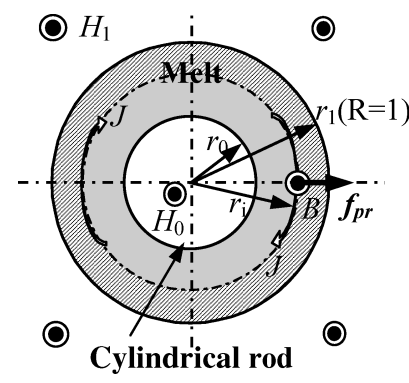

Fig. 3. Electromagnetic force exerted on the cross profile of hollow cylindrical melt.

of the melt and $f$ is the frequency of the magnetic field, the expressions of the vectors $\dot{\mathbf{J}}_{r}$ and $\dot{\mathbf{H}}_{r}$ can be derived as:

$$
\begin{gathered}
\dot{\mathbf{H}}_{r}=H_{1} \frac{b e r \xi R+i b e i \xi R}{b e r \xi+i b e i \xi} \ldots \ldots \ldots \ldots . . . \\
\dot{\mathbf{J}}_{r}=-\frac{d H_{r}}{d r}=-\frac{\xi}{r_{1}} H_{1} \frac{b e r^{\prime} \xi R+i b e i^{\prime} \xi R}{b e r \xi+i b e i \xi}
\end{gathered}
$$

where $H_{r}$ is the applied magnetic intensity at the radial distance $r, H_{1}$ is the magnetic intensity at the exterior surface, ber and bei represent 0th Kelvin function, the nondimensional factor $\xi=\sqrt{2} r_{1} / \delta, R=r / r_{1}$, and $r_{1}$ is the radius of the solid cylinder.

By substituting Eqs. (5) and (6) into Eq. (3), electromagnetic force exerted on a unit volume of the melt can be derived as:

$$
\begin{array}{r}
f_{r}=-\frac{B_{e}^{2}}{\sqrt{2} \mu_{e} r_{1}} f^{*}(\xi, R) \ldots \ldots \ldots \ldots \ldots \ldots \ldots . . .(7) \\
f^{*}(\xi, R)=\xi \frac{b e r \xi R \cdot\left(b e r_{1} \xi R+b e i_{1} \xi R\right)-b e i \xi R\left(b e r_{1} \xi R-b e i_{1} \xi R\right)}{b e r^{2} \xi+b e i^{2} \xi}
\end{array}
$$

where $B_{e}$ is the effective magnetic flux density, $B_{e}=$ $\left(\mu_{e} / \sqrt{2}\right) H_{1}$, ber $_{1}$ and $b e i_{1}$ represent 1st Kelvin function.

According to the Stokes Law, the terminal migration velocity of spherical particles $v_{p r}$ can be derived as ${ }^{8}$ :

$$
v_{p r}=-\frac{d_{p}^{2} B_{e}^{2}}{24 \sqrt{2} \mu \mu_{e} r_{1}} f^{*}(\xi, R)
$$

where $\mu$ is the kinetic viscosity of the melt.

\section{Theoretical Calculation in the Hollow Cylindrical Melt Case}

\subsection{Assumptions}

Supposing a cylindrical rod made of refractory material is placed at the center of the melt, as shown in Fig. 3, we obtain a hollow cylindrical melt. Apparently, the solid cylindrical melt is one of special cases of the hollow cylindrical melt while the internal radius of the melt $r_{0}$ is zero.

In order to simplify the theoretical calculation, the following assumptions are made firstly:

(1) The magnetic field and the induced current are constant 
along the axial direction;

(2) The magnetic flux density is homogenous at the exterior surface of the melt along the circumferential direction;

(3) The inclusion particles are nonconductive and spherical in shape;

(4) The inclusion particles is evenly distributed in the melt;

(5) The density of the particle is uniform and consistent with the melt, namely the buoyancy can be neglected;

(6) The particles are so small that their inertia is negligible, and their migration in the melt follow the Stokes Law;

(7) The particles are captured immediately when arriving at the sidewall.

\subsection{Calculation Results}

3.2.1. Electromagnetic Force and Migration Velocity

The imposed alternating magnetic field can be expressed by ${ }^{12)}$ :

$$
H=H_{0} e^{j \omega t}
$$

where $\omega$ is the angular frequency, $H_{0}$ is the magnetic intensity at the interior surface.

Similar with cylindrical case, the solution of Eq. (10) derived by solving Eq. (4) have a form expressed by Bessel functions ${ }^{13)}$ :

$$
H_{r}=A I_{0}(k r)+B K_{0}(k r)
$$

where $H_{r}$ is the magnetic intensity in the area of $r_{0} \leq r \leq r_{1}$; $A$ and $B$ are constants; $k=(1+\mathrm{i}) / \delta ; I_{0}$ and $K_{0}$ are zerothorder Bessel functions.

According to the boundary conditions at the place where $r=r_{0}$ and $r=r_{1}$ :

$$
\left\{\begin{array}{l}
H_{0}=A I_{0}\left(k r_{0}\right)+B K_{0}\left(k r_{0}\right) \\
H_{1}=A I_{0}\left(k r_{1}\right)+B K_{0}\left(k r_{1}\right)
\end{array}\right.
$$

where $r_{0}$ and $r_{1}$ are the internal and external radius of the cylinder respectively, the final solutions of Eq. (10), $\dot{\mathbf{J}}_{r}$ and $\dot{\mathbf{H}}_{r}$ can be derived as follows:

$$
\begin{array}{r}
\dot{\mathbf{H}}_{r}=H_{1}\left[\frac{c_{r}+i c_{i}}{d_{r}+i d_{i}} I_{0}(\xi R)+\frac{K_{0}(\xi R)}{d_{r}+i d_{i}}\right] \ldots \ldots \ldots . .(13) \\
\dot{J}_{r}=-\left(\frac{\partial H}{\partial r}\right)=-\frac{\sqrt{2} H_{1}}{\left(d_{r}+i d_{i}\right) \cdot \delta}\left[\left(c_{r}+i \cdot c_{i}\right) I_{0}{ }^{\prime}(\xi R)+K_{0}{ }^{\prime}(\xi R)\right]
\end{array}
$$

where $c_{r}, c_{i}, d_{r}, d_{i}$ are factors in relation with $r_{0}, \delta$ and Kelvin functions ber, bei, ker and kei. The definitions of $c_{r}$, $c_{i}, d_{r}$ and $d_{i}$ are expressed as follows ${ }^{13)}$ :

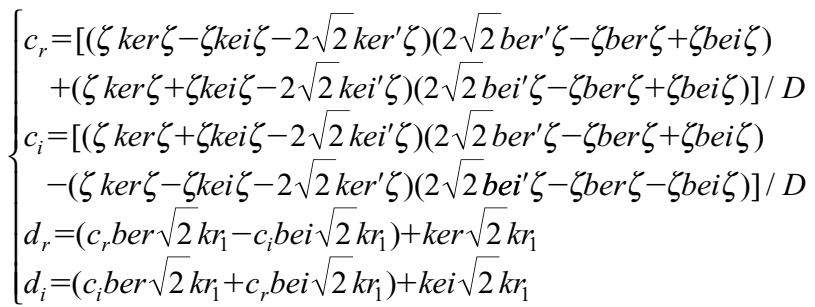

where the nondimensional factor $\zeta=\sqrt{2} r_{0} / \delta$ and

$$
\begin{aligned}
D= & \left(\sqrt{2} \text { ber }^{\prime} \zeta-\text { ber } \zeta+\zeta \text { bei } \zeta\right)^{2} \\
& +(2 \sqrt{2} \text { bei' }-\zeta \text { ber } \zeta-\zeta b e i \zeta)^{2}
\end{aligned}
$$

Similar to the solid cylinder case, by substituting Eqs. (13) and (14) into Eq. (3), the electromagnetic pinch force can be obtained as:

$$
f_{r}=-\frac{B_{e}^{2}}{\sqrt{2} \mu_{e} r_{1}} f_{h}^{*}(\xi, R)
$$

where

$$
\begin{gathered}
f_{h}^{*}(\xi, R)=\xi \cdot \frac{P(\xi, R) \cdot M(\xi, R)+Q(\xi, R) \cdot N(\xi, R)}{d_{r}^{2}+d_{i}^{2}} \\
\left\{\begin{array}{r}
P(\xi, R)=d_{r} \cdot\left(\operatorname{ber} \xi R \cdot c_{r}-\text { bei } \xi R \cdot c_{i}+k e r \xi R\right) \\
+d_{i} \cdot\left(\operatorname{ber} \zeta R \cdot c_{i}+b e i \xi R \cdot c_{r}+k e i \xi R\right) \\
Q(\xi, R)=d_{r} \cdot\left(\text { ber } \xi R \cdot c_{i}+b e i \xi R \cdot c_{r}+k e i \xi R\right) \\
-d_{i} \cdot\left(\operatorname{ber} \xi R \cdot c_{r}-b e i \xi R \cdot c_{i}+k e r \xi R\right) \\
M(\xi, R)=m(\xi, R) \cdot d_{r}+n(\xi, R) \cdot d_{i} \\
N(\xi, R)=n(\xi, R) \cdot d_{r}-m(\xi, R) \cdot d_{i}
\end{array}\right.
\end{gathered}
$$

where

$$
\left\{\begin{aligned}
m(\xi, R)= & \operatorname{ber}^{\prime} \xi R \cdot c_{r}-\operatorname{bei}^{\prime} \xi R \cdot c_{i}+k e r^{\prime} \xi R \\
& -\operatorname{ber}^{\prime} \xi R \cdot c_{i}-b e i^{\prime} \xi R \cdot c_{r}-\operatorname{kei}^{\prime} \xi R \\
n(\xi, R)= & \operatorname{ber}^{\prime} \xi R \cdot c_{r}-\operatorname{bei}^{\prime} \xi R \cdot c_{i}+k e r^{\prime} \xi R \\
& +\operatorname{ber}^{\prime} \xi R \cdot c_{i}+\operatorname{bei}^{\prime} \xi R \cdot c_{r}+k e i^{\prime} \xi R
\end{aligned}\right.
$$

According to the Stokes Law, the terminal migration velocity of spherical particles $v_{p r}$ can be expressed as follows:

$$
v_{p r}=-\frac{d_{p}^{2}}{24 \mu} \cdot f_{r}
$$

where $\mu$ is the kinetic viscosity of the melt.

By substituting Eq. (17) into Eq. (21), the migration velocity in the hollow cylindrical melt case can be obtained as follows:

$$
v_{p r}=-\frac{d_{p}^{2} B_{e}^{2}}{24 \sqrt{2} \mu \mu_{e} r_{1}} f_{h}^{*}(\xi, R)
$$

3.2.2. Comparison between Hollow Cylinder and Solid Cylinder Case

Comparing Eq. (9) with Eq. (20), it is clear that the migration velocity in the two cases depend on the functions $f^{*}(\xi, R)$ and $f_{h}^{*}(\xi, R)$, respectively, in respect to the constant value of $B_{e}, \mu_{e}$ and $r_{1}$.

The relation between $f^{*}(\xi, R), f_{h}^{*}(\xi, R)$ and the nondimensional factor $R$ are shown in Figs. $\mathbf{4}$ and 5, respectively, where parameter $\lambda=r_{0} / r_{1}, r_{0}$ and $r_{1}$ is the internal radius and external radius of the hollow cylinder, respectively. Apparently, the value of $f^{*}(\xi, R)$ is very small in most of the area $(R<0.8)$. Therefore, the removal efficiency of inclusion particles can hardly be improved by changing magnetic processing parameters because most of the particles are located in the central area far from the skin depth layer and 


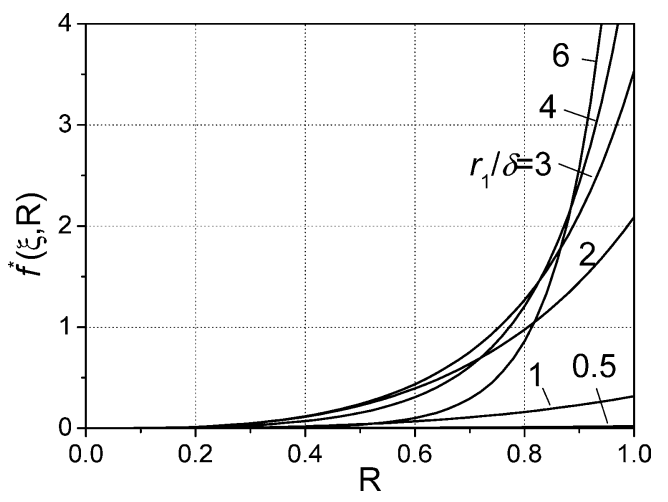

Fig. 4. Calculated values of $f^{*}(\xi, R)$ as a function of $R$.

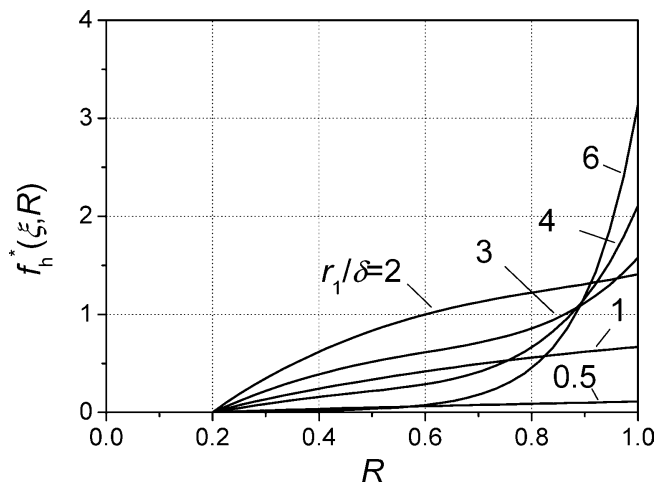

Fig. 5. Calculated values of $f_{h}^{*}(\xi, R)$ as a function of $R$ at $\lambda=0.2$.

less influenced by the magnetic field. However, $f_{h}^{*}(\xi, R)-R$ curve of hollow cylinder case appears to be convex shape if proper value of the non-dimensional parameter $r_{1} / \delta$ is selected in the range from 1 to 3 . Compared with the solid cylinder case, the electromagnetic forces exerted on the particles located in the interior area of the melt are increased remarkably in the hollow cylinder case. Accordingly, the removal efficiency may be improved by using hollow cylindrical melt instead of solid cylindrical melt.

\subsubsection{Removal Efficiency of Particles}

Reference 9) proposed a special method to calculate the removal efficiency of inclusion particles. Due to the imposed electromagnetic force, a spherical particle will experience a varying accelerated motion procedure and migrate from the original position $R_{i}$ to the outer wall where $R=1$, as shown in Figs. 2 and 3. The time when it arrives at the wall $t_{i}$, can be expressed as:

$$
t_{i}=r_{1} \cdot \int_{R_{i}}^{1} \frac{1}{v_{p r}} d R
$$

By the time when $t=t_{i}$, all the particles located at the striated areas in Figs. 2 and 3 where $R>R_{i}\left(r>r_{i}\right)$ will be moved to the wall and captured. Therefore, the corresponding removal efficiency in the two cases can be defined as follows, respectively:

Solid cylinder case: $\quad \eta=1-R_{i}^{2}$

Hollow cylinder case: $\quad \eta=\frac{1-R_{i}^{2}}{1-\lambda^{2}}$

where $\lambda=r_{0} / r_{1}$.

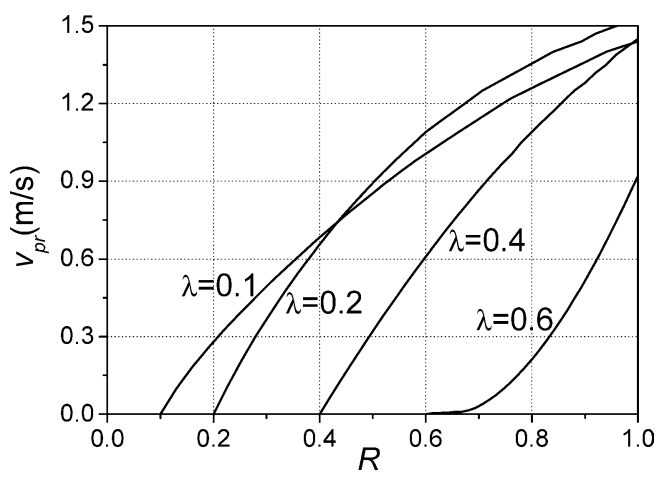

Fig. 6. Effect of $\lambda$ on the relationship between $v_{p r}$ and $R$ $\left(r_{1}=10 \mathrm{~mm}, d_{p}=10 \mu \mathrm{m}, B_{e}=0.1 \mathrm{~T}, \delta=r_{1}-r_{0}\right)$.

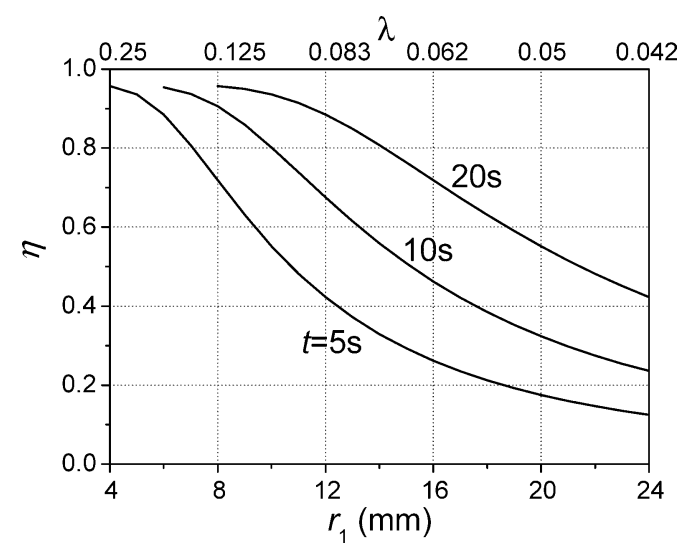

Fig. 7. Relation between $\eta$ and $r_{1}$ at different imposing time $\left(d_{p}=10 \mu \mathrm{m}, r_{0}=1 \mathrm{~mm}, B_{e}=0.1 \mathrm{~T}, r_{1} / \delta=2\right)$

According to Eqs. (23), (24) and (25), the relation between parameters and the removal efficiency can be deduced. The following discusses will focus mainly on the effects of various parameters on removal efficiency in hollow cylinder case.

\subsection{Effects of Various Parameters on Removal Effi- ciency}

3.3.1. Nondimensional Parameter $\lambda$

The geometry of the hollow cylindrical melt is determined by $\lambda$ and $r_{1}$. In our calculations four different values of $\lambda$ were selected while the skin depth, $\delta$ is fixed to be equal to the wall thickness of the hollow cylinder, namely $\delta=r_{1}-r_{0}$, and the external radius is fixed to be $r_{1}=10 \mathrm{~mm}$. The relationship between $v_{p r}$ and $R$ at $d_{p}=10 \mu \mathrm{m}$ and $B_{e}=0.1 \mathrm{~T}$ is given in Fig. 6. It can be seen that higher migration velocity of the particles can be obtained when the value of $\lambda$ is selected between 0.1 and 0.4 . On the other hand, nondimensional parameter $\lambda$ decreases with the increasing value of $r_{1}$ while the value of $r_{0}$ is fixed. Accordingly, the removal efficiency $\eta$ decreases with decreasing value of $\lambda$ below 0.2 , as shown in Fig. 7 . Thus, it can be easily concluded that the proper values of $\lambda$ should be selected as about 0.2 to obtain higher removal efficiency.

\subsubsection{Nondimensional Parameter $r_{1} / \delta$}

Firstly, we evaluate the effect of $r_{1} / \delta$ on the removal efficiency when the value of $r_{1}$ is constant and is set as 0.2 . Figure 8 shows the relation between $\eta$ and $r_{1} / \delta$ at $B_{e}=0.1 \mathrm{~T}$ and $t=10 \mathrm{~s}$. As seen from the figure, the value of $\eta$ approaches maximum when $r_{1} / \delta$ is in the range of $1.5-2$. 


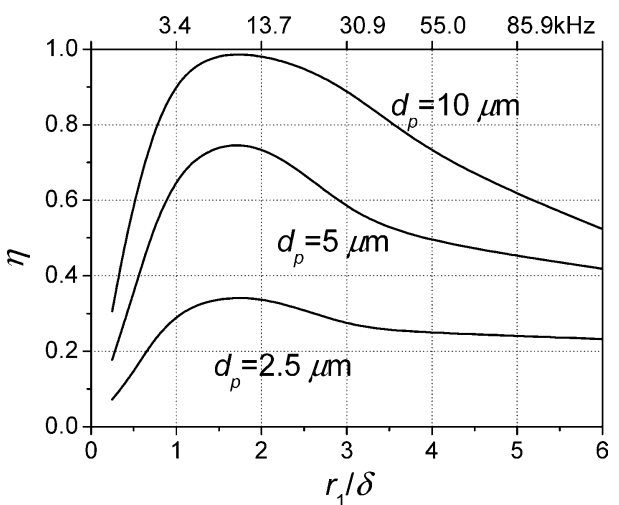

Fig. 8. Relation between $\eta$ and $r_{1} / \delta$ with constant value of $r_{1}$ $\left(r_{1}=10 \mathrm{~mm}, t=10 \mathrm{~s}, \lambda=0.2, \mathrm{Be}=0.1 \mathrm{~T}\right)$.

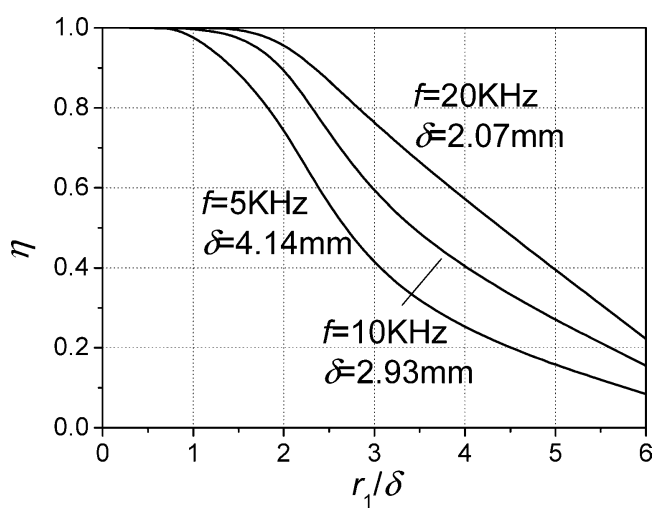

Fig. 9. Relation between $\eta$ and $r_{1} / \delta$ with constant frequency $\left(d_{p}=10 \mu \mathrm{m}, t=10 \mathrm{~s}, r_{0}=1 \mathrm{~mm}, B_{e}=0.1 \mathrm{~T}\right)$

For example, higher value of $\eta$ approaching 100 pct can be obtained for the particles larger than $10 \mu \mathrm{m}$. In addition, the value of $d_{p}$ influences the removal efficiency remarkably. The maximal value of $\eta$ decreases rapidly with decreasing value of $d_{p}$. Especially for the particles with $2.5 \mu \mathrm{m}$ diameters, the maximum of $\eta$ only approaches $35 \%$. When the value of $r_{1} / \delta$ ranges below 1.5 , the corresponding frequency is smaller than $8 \mathrm{KHz}$, as shown in Fig. 8. Obviously, the electromagnetic force inclines to be relatively uniform and weak in the whole range of the melt so that it is very difficult to increase the removal efficiency. On the other hand, the value of $\delta$ will be smaller than $2.5 \mathrm{~mm}$ while the value of $r_{1} / \delta$ ranges above 2 at the corresponding value of $f$ larger than $15 \mathrm{kHz}$. It also leads to lower removal efficiency for the reason that the proportion of the skin layer to the whole cross area becomes smaller with decreasing value of the skin depth.

Secondly, the effect of $r_{1} / \delta$ on the removal efficiency with constant frequency and various $r_{1}$ is also evaluated in Fig. 9, where the value of $r_{1} / \delta$ ranges from zero to 6 . It can be seen from the figure that the value of $\eta$ decreases with the increase of $r_{1}$. Moreover, the lower frequency and larger skin depth, the faster decreasing trend of $\eta$ will be. Apparently, the proportion of the skin layer to the whole melt also turns to be smaller with increasing value of $r_{1}$ and it leads to weaker electromagnetic force exerted on the particles. In the case while $r_{1}>>r_{0}$, it approximates to the solid cylinder case. And if the frequency is set as above $10 \mathrm{kHz}$, the optimal value of $r_{1} / \delta$ should be no more than 2 .

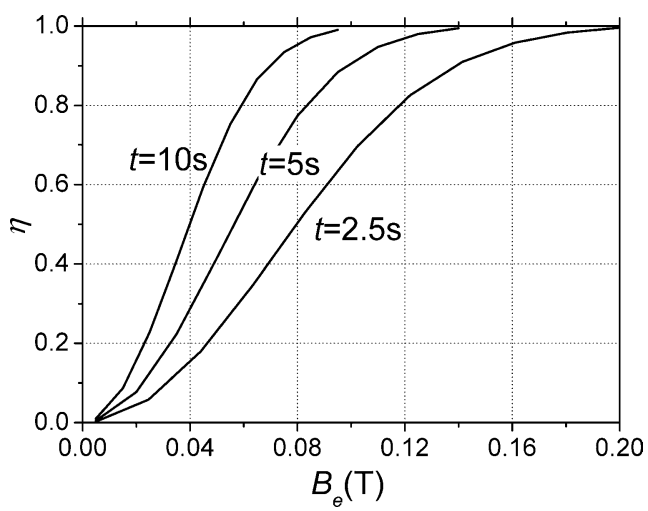

Fig. 10. Effect of $t$ on the relationship between $\eta$ and $B_{e}$ $\left(r_{1}=10 \mathrm{~mm}, d_{p}=10 \mu \mathrm{m}, \lambda=0.2, r_{1} / \delta=2\right)$

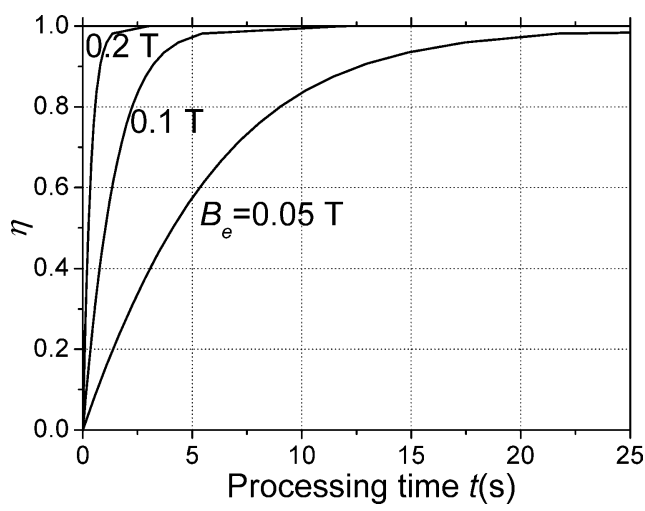

Fig. 11. Effect of $B_{e}$ on the relationship between $\eta$ and $t$ $\left(r_{1}=10 \mathrm{~mm}, d_{p}=10 \mu \mathrm{m}, \lambda=0.2, r_{1} / \delta=2\right)$.

According to the above analysis, it can be concluded that the value of $\eta$ exceed $90 \%$ for the $10 \mu \mathrm{m}$ particles under the conditions that the value of $r_{1} / \delta$ ranges from 1.5 to 2 and the frequency ranges from 10 to $15 \mathrm{KHz}$.

\subsubsection{Imposing Time $t$}

Figure 10 illustrates the relation between the removal efficiency and the magnetic flux density while parameter $\lambda$ is set as 0.2 and the imposing time is set as $2.5 \mathrm{~s}, 5 \mathrm{~s}$ and $10 \mathrm{~s}$, respectively. It can be seen that the removal efficiency is too low while the value of $B_{e}$ is below $0.1 \mathrm{~T}$ and the imposing time is no more than $5 \mathrm{~s}$. Therefore, the minimum imposing time must be set as more than $5 \mathrm{~s}$ at $B_{e}=0.1 \mathrm{~T}$ for efficient removal of $10-\mu \mathrm{m}$ particles.

\subsubsection{Magnetic Flux Density $B_{e}$}

Since the electromagnetic force is proportional to the square of the magnetic flux density, as shown in Eq. (12), it is clear that $B_{e}$ is a very important processing parameter for electromagnetic separation. Figure 11 shows the effect of $B_{e}$ on the relationship between removal efficiency and the imposing time. As seen from the figure, the removal efficiency is rather low in the range of $t \leq 10 \mathrm{~s}$ while the value of $B_{e}$ is set as $0.05 \mathrm{~T}$. However, the removal efficiency increases rapidly with the increasing $\mathrm{t}$ while the value of $B_{e}$ is larger than $0.1 \mathrm{~T}$. And the value of $\eta$ exceeds $90 \%$ after processing $5 \mathrm{~s}$ at $0.1 \mathrm{~T}$. The above analysis indicates that the removal efficiency strongly depends on the magnetic flux density. Therefore, in order to efficiently remove small particles, the value of $B_{e}$ should be selected higher than $0.1 \mathrm{~T}$ 




Fig. 12. Relation between $\eta$ and $d_{p} \quad\left(r_{1}=10 \mathrm{~mm}, \lambda=0.2\right.$, $\left.B_{e}=0.1 \mathrm{~T}, r_{1} / \delta=2\right)$

in general.

\subsubsection{Particle Diameter $d_{p}$}

The particle velocity increases with the square of the increase of particle diameter $d_{p}$, as shown in Eq. (16). The dependence of $\eta$ upon $d_{p}$ in solid cylinder and hollow cylinder cases is illustrated in Fig. 12 respectively, which shows that the value of $\eta$ decreases rapidly with decreasing value of $d_{p}$ below $5 \mu \mathrm{m}$. However, the value of $\eta$ is much higher in the hollow cylindrical melt case than that of solid cylinder case, especially when the value of $d_{p}$ is above $2.5 \mu \mathrm{m}$. In other words, it is more efficient to separate micro-sized particles in hollow cylinder case than in solid cylinder case.

\section{Conclusions}

(1) Compared with the solid cylinder case, the electromagnetic forces exerted on the particles located in the interior area of the melt are increased remarkably in the hollow cylinder case. Therefore, the removal efficiency can be improved by using hollow cylindrical melt instead of solid cylindrical melt.

(2) The inclusion particles larger than $5 \mu \mathrm{m}$ can be removed easily in the hollow cylindrical case and the removal efficiency will exceed 90 pct with proper processing parameters.

(3) To obtain higher removal efficiency, proper values of $f, \lambda$, and $r_{1} / \delta$ should be selected as $10-15 \mathrm{KHz}$, about 0.2 , and $1.5-2$, respectively. Moreover, the optimal numeric range of other processing parameters is that $B_{e}>0.1 \mathrm{~T}$ and $t>5 \mathrm{~s}$.

\section{Acknowledgments}

Financial supports from the High Technology Research and Development Program of China (Grant No. 2002AA336072), the National Natural Science Foundation of China (Grant No. 50101005) and the National Key Research Foundation of China (Grant No.G1999064900-4) are gratefully acknowledged.

\section{Nomenclature}

B: Magnetic flux density vector $(\mathrm{T})$

$B_{e}: \quad$ Effective magnetic flux density $(\mathrm{T})$

$c_{r}, c_{i}, d_{r}, d_{i}$ : Factors in relation with $r_{0}, \delta$ and Kelvin functions ber, bei, ker and kei

$b_{n}, b e i_{n}$ : The $n$th order Kelvin functions $d$ : Diameter of ceramic pipes ( $\mathrm{mm})$

$d_{p}: \quad$ Diameter of particles $(\mu \mathrm{m})$

$f$ : $\quad$ Frequency of magnetic field $(\mathrm{Hz})$

$\mathbf{f}_{r}$ : Electromagnetic pinch force acting on a unit volume of the melt $\left(\mathrm{N} / \mathrm{m}^{2}\right)$

$f_{r}$ : Root-mean-square value of $\mathbf{f}_{r}\left(\mathrm{~N} / \mathrm{m}^{2}\right)$

$\mathbf{f}_{p r}$ : Electromagnetic force acting on a unit volume of particles $(\mathrm{N})$

$H$ : Applied magnetic intensity $(\mathrm{A} / \mathrm{m})$

$H_{0}$ : Applied magnetic intensity at the interior surface of the hollow cylinder melt $(\mathrm{A} / \mathrm{m})$

$H_{1}$ : Applied magnetic intensity at the exterior surface of the hollow cylinder melt $(\mathrm{A} / \mathrm{m})$

$H_{r}$ : Applied magnetic intensity at the radial distance $r(\mathrm{~A} / \mathrm{m})$

$\dot{\mathbf{H}}_{r}$ : Applied magnetic intensity vector at the radial distance $r(\mathrm{~A} / \mathrm{m})$

$I_{0}, K_{0}$ : The zero-th-order Bessel functions

$\mathbf{J}$ : Induced electric current density vector $\left(\mathrm{A} / \mathrm{m}^{2}\right)$

$\dot{\mathbf{J}}_{r}$ : Induced current density vector at the radial distance $r\left(\mathrm{~A} / \mathrm{m}^{2}\right)$

$k$ : Constant

$R$ : Nondimensional radial distance $R=r / r_{1}$

$r$ : Radial distance $(\mathrm{m})$

$r_{0}, r_{1}$ : Internal and external radius of the cylinder, respectively $(\mathrm{m})$

$t$ : Processing time (s)

$v_{p r}$ : Terminal radial migration rate of particle $(\mathrm{m} / \mathrm{s})$

$\mu: \quad$ Kinetic viscosity of melt $(\mathrm{Pa} \cdot \mathrm{s})$

$\mu_{e}: \quad$ Magnetic permeability of melt $(\mathrm{H} / \mathrm{m})$

$\eta$ : Removal efficiency of particles

$\alpha: \quad \alpha=(1+i) / \delta$

$\delta: \quad$ Skin depth (m) $\delta=\sqrt{1 / \pi \mu_{e} \sigma_{f} f}$

$\sigma_{f}: \quad$ Electrical conductivity of melt $(\mathrm{S} / \mathrm{m})$

$\xi$ : Nondimentional factor $\xi=\sqrt{2} r_{1} / \delta$

$\zeta$ : Nondimentional factor $\zeta=\sqrt{2} r_{0} / \delta$

$\omega$ : Angular frequency $(\mathrm{rad} / \mathrm{s})$

$\lambda$ : Nondimensional factor, $\lambda=r_{0} / r_{1}$

\section{REFERENCES}

1) R. D. Peterson: Proc. of 3rd Int. Conf. on Aluminum Processing, American Foundrymen's Society, Des Plaines. IL, (1994), 75.

2) A. R. Robert: JOM, 49 (1997), 21.

3) P. Marty and A. Alemany: Proc. of a Symp. of the IUTAM, The Metals Society, London, (1982), 245.

4) V. M. Korovin: Magnetiohydrodynamics, 21 (1985), 321.

5) V. M. Korovin: Magnetiohydrodynamics, 22 (1986), 17.

6) D. Shu, B. D. Sun, J. Wang, T. X. Li and Y. H. Zhou: Metall. Mater. Trans. A, 30A (1999), 2979.

7) K. Li, B. D. Sun, D. Shu, T. X. Li and Y. H. Zhou: Acta Metall. Sin., 37 (2001), 405

8) F. Yamao, K. Sassa, K. Iwai and S. Asai: Tetsu-to-Hagané, 83 (1997), 30.

9) S. Taniguchi and J. K. Brimacombe: ISIJ Int., 34 (1994), 722.

10) N. El-Kaddah, A. D. Patel and T. T. Natarajan: JOM, 47 (1995), 46.

11) N. El-Kaddah: Conference Records of IEEE on Industrial Applications, (1988), 1162

12) J. Davies and P. Simpson: Induced Heating Handbook, McGraw-Hill, New York, (1979), 117

13) D. P. Xi: Functions of Bessel, High educational publishing house, Beijing, (1998), 310. 